\title{
Erratum: Efficient Generation of Model Bulk Heterojunction Morphologies for Organic Photovoltaic Device Modeling [Phys. Rev. Applied 2, 014008 (2014)]
}

\author{
Michael C. Heiber and Ali Dhinojwala \\ (Received 14 April 2017; published 20 July 2017)
}

DOI: 10.1103/PhysRevApplied.8.019902

The computational software tool that we have developed and used in the original paper has been found to contain a bug that significantly affects some of the results of the study. We report here updated results from the corrected software tool.

The phase separation process in the previously presented Ising-based morphology model proceeds by a series of siteswapping attempts, where neighboring sites of opposing types are swapped with a probability that depends on the interaction energy $(J)$. In v1.0 and v2.0 of the IsING_OPV morphology generation tool [1,2], there was a bug in the code that affected how the pair of sites was randomly selected before undergoing a swap attempt. This systematic error caused anisotropic domain growth during phase separation. In effect, the domains had a slight preference for growing in the $x$ and/or $y$ directions of the lattice (parallel to the plane of the film) relative to the $z$ direction (film thickness direction). This bug was identified upon the addition of a domain anisotropy characterization feature in v3.0 of the ISING_OPV software tool [3]. In a system with an isotropic lattice size and interactions, the morphologies should have no anisotropy.

With this error now corrected in ISING_OPV v3.0, we retest the impact that the interaction energy $(J)$ has on the domain growth kinetics and the resulting interfacial-area-to-volume ratio and tortuosity. For each morphology set generated, the domain structure is confirmed to be isotropic as expected. Figure 1 shows how decreasing the interaction energy still greatly increases the rate at which domains grow in the system, in agreement with our previous results. In fact, the growth kinetics are actually slightly faster with the corrected code.

We previously found that the interaction energy affects the interfacial-area-to-volume ratio of the final morphologies, especially when the domain size is greater than $7 \mathrm{~nm}$. However, in the results from the corrected code shown in Fig. 2, we find no significant difference between the morphologies generated with different interaction energies. Only the smallest interaction energy of $0.3 \mathrm{kT}$ shows some deviation for 6-nm domains.

We also previously found that differences in the interfacial-area-to-volume ratio reflected changes in the domain shape and/or connectivity and that this behavior could be most clearly identified by changes in the tortuosity of the chargetransport pathways through the film. However, the corrected results shown in Fig. 3 demonstrate that the interaction energy has no meaningful impact on the tortuosity. These updated results suggest that the tortuosity differences observed in our previous study were due to the anisotropic domain growth artifact. In our updated tests, the lack of domain anisotropy results in morphologies with a low tortuosity that is independent of domain size. This correction indicates that when using the domain-smoothing algorithm we previously developed, the morphologies generated using the standard Ising-based model do not significantly differ when created using interaction energies in the range of 1.0 down to $0.4 \mathrm{kT}$.

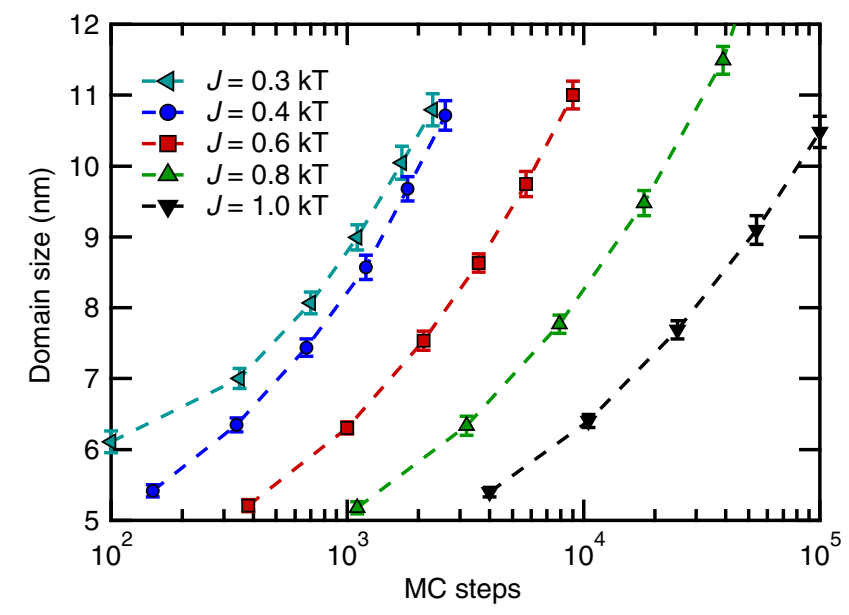

FIG. 1. Growth kinetics for different values of the interaction energy $(J)$. 


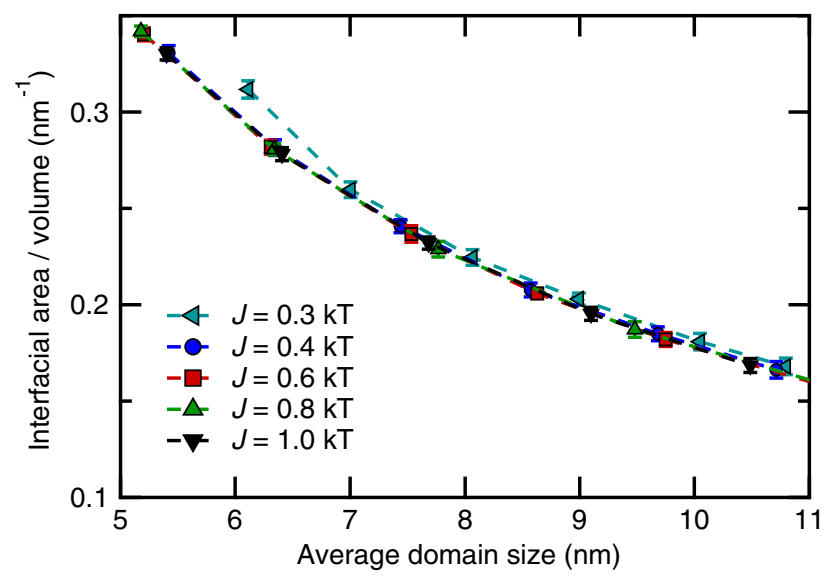

FIG. 2. Interfacial-area-to-volume ratio values for morphology sets with varying interaction energy $(J)$ and domain size.

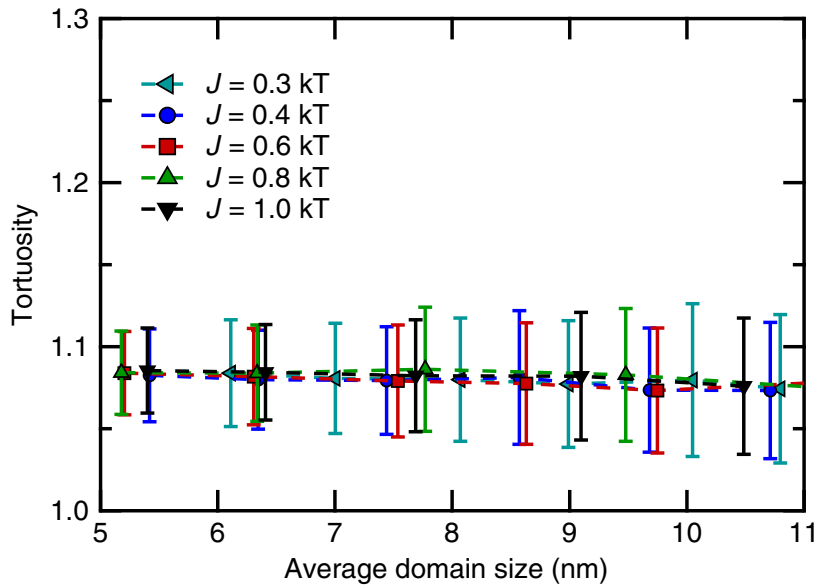

FIG. 3. Tortuosity values for morphology sets with varying interaction energy $(J)$ and domain size.

With the corrected code, we also test the lattice size restrictions as shown in Fig. 4 and find that when the lattice size is smaller than about 3.8 times the domain size, the finite size of the lattice has an impact on the final domain size. This value is slightly smaller than what we determined in the previous study, which is due to the domain anisotropy that was present in the previous morphologies.

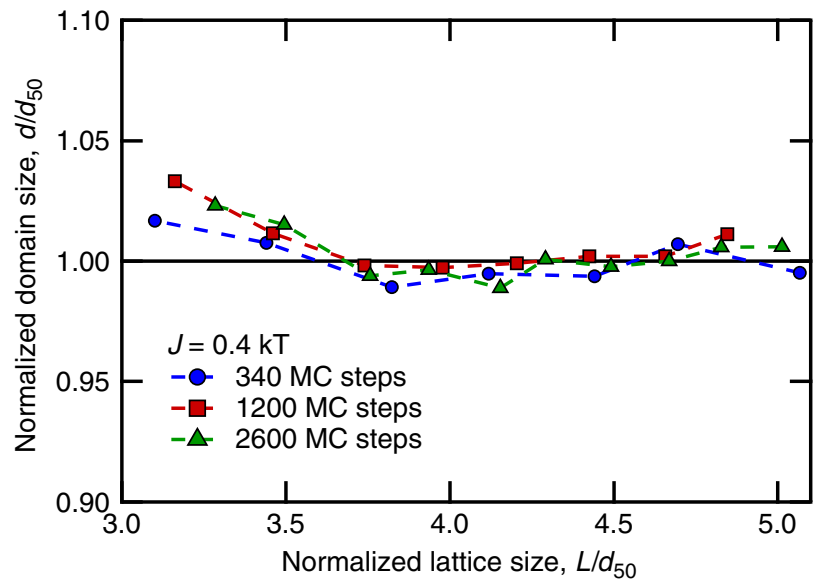

FIG. 4. The effect that a finite lattice size has on the final domain size. 
Overall, these updated results show that with the Ising-based morphology model, the interaction energy does not affect the tortuosity of the morphology. In addition, we find that it is safe to use an interaction energy as small as $0.4 \mathrm{kT}$ to quickly generate morphologies that are equivalent to those created much more slowly with larger interaction energies. This allows for an even more computationally efficient generation of model morphologies for bulk heterojunction organic photovoltaic simulations. In total, this development reduces the required number of iterations by over 1 order of magnitude, which concomitantly reduces the calculation time by a similar magnitude. These developments continue to allow larger and more complex morphologies to be investigated in pursuit of more detailed and accurate models for the structure-property relationship in organic photovoltaic blends $[4,5]$.

This work uses the Extreme Science and Engineering Discovery Environment (XSEDE) for computing resources [6], which is supported by National Science Foundation Grant No. ACI-1053575, and M. C. H. acknowledges the XSEDE allocation Grant No. TG-DMR150118.

[1] M. C. Heiber, Ising_OPV v1.0, https://github.com/MikeHeiber/Ising_OPV, 2014.

[2] M. C. Heiber, Ising_OPV v2.0, https://github.com/MikeHeiber/Ising_OPV, 2015.

[3] M. C. Heiber, Ising_OPV v3.0, https://github.com/MikeHeiber/Ising_OPV, 2016.

[4] M. C. Heiber, C. Baumbach, V. Dyakonov, and C. Deibel, Encounter-Limited Charge-Carrier Recombination in Phase-Separated Organic Semiconductor Blends, Phys. Rev. Lett. 114, 136602 (2015).

[5] M. C. Heiber, T.-Q. Nguyen, and C. Deibel, Charge carrier concentration dependence of encounter-limited bimolecular recombination in phase-separated organic semiconductor blends, Phys. Rev. B 93, 205204 (2016).

[6] J. Towns, T. Cockerill, M. Dahan, I. Foster, K. Gaither, A. Grimshaw, V. Hazlewood, S. Lathrop, D. Lifka, G. D. Peterson, R. Roskies, J. R. Scott, and N. Wilkins-Diehr, XSEDE: Accelerating scientific discovery, Comput. Sci. Eng. 16, 62 (2014). 